\title{
Sirenomelia associada a defeitos congênitos raros: relato de três casos
}

Primeira submissão em 23/01/12 Última submissão em 16/05/12 Aceito para publicação em $27 / 05 / 12$ Publicado em 20/08/12

\section{Sirenomelia associated with rare congenital defects: report of three cases}

Maria Angélica F. D. Lima'; Heloisa Novaes Machado²; Dione Correa de Araújo Dock; Maria Auxiliadora Monteiro Villar'; Juan Clinton Llerena Junior ${ }^{5}$

\begin{tabular}{l|l}
\multicolumn{1}{c|}{ unitermos } & resumo \\
\hline Sirenomelia & Sirenomelia é um defeito congênito muito raro do campo primário do desenvolvimento, definido \\
Malformação & pela substituição dos membros inferiores, normalmente pareados por um único membro mediano. \\
Patologia & $\begin{array}{l}\text { Geralmente, associa-se a graus variados de anomalias gênito-urinárias. Relatamos três casos necropsiados } \\
\text { dessa entidade, incluindo estudo radiológico do membro inferior único, associados a agenesia renal } \\
\text { bilateral, de ureteres e da bexiga, atresia retal, ânus imperfurado, testículos abdominais e ausência } \\
\text { de genitália externa, além de outros defeitos congênitos infrequentemente observados, que somente } \\
\text { puderam ter seus diagnósticos firmados por meio da necropsia. }\end{array}$
\end{tabular}

abstract

Sirenomelia, an extremely rare congenital defect, is defined as a limb abnormality in which the normally paired lower limbs are replaced by a single midline limb. It is commonly associated with varied genitourinary anomalies. We report three cases of sirenomelia including x-ray documentation of the lower limb. Other associated aspects, whose diagnoses were established exclusively through autopsy, included bilateral renal, ureteral and bladder agenesis, rectal atresia, imperforate anus, intra-abdominal testis, absence of external genitalia and other rare congenital abnormalities. key words

Sirenomelia

Malformation

Pathology

\footnotetext{
1. Mestra em Ciências; médica do Instituto Nacional de Saúde da Mulher, da Criança e do Adolescente Fernandes Figueira/Fundação Oswaldo Cruz (FIOCRUZ).

2. Doutora em Ciências; médica do Departamento de Anatomia Patológica do Instituto Nacional da Saúde da Mulher, da Criança e do Adolescente Fernandes Figueira/FIOCRUZ.

3. Especialista em Anatomia Patológica; médica do Departamento de Anatomia Patológica do Instituto Nacional da Saúde da Mulher, da Criança e do Adolescente Fernandes Figueira/FIOCRUZ.

4. Doutora em Ciências; médica do Instituto Nacional de Saúde da Mulher, da Criança e do Adolescente Fernandes Figueira/FIOCRUZ; pediatra do Departamento de Cenética Médica do Instituto Nacional de Saúde da Mulher, da Criança e do Adolescente Fernandes Figueira/FIOCRUZ.

5. Doutor em Genética pela Universidade Federal do Rio de Janeiro (UFR)); chefe do Departamento de Genética Médica do Instituto Nacional de Saúde da Mulher, da Criança e do Adolescente Fernandes Figueira/FIOCRUZ.
} 


\section{Introdução}

Sirenomelia é um defeito congênito raro com prevalência estimada de 0,98:100.000 nascimentos ${ }^{(7,13)}$. É definida como defeito caracterizado pela substituição dos membros inferiores, normalmente pareados por um único membro mediano ${ }^{(8,13)}$. Ocorre em torno da terceira semana de vida intrauterina ${ }^{(12)}$ e associa-se a anomalias gênito-urinárias, incluindo agenesia renal, de ureteres e da bexiga, ânus imperfurado e ausência da genitália externa ${ }^{(15)}$, consideradas malformações graves, levando à morte no período perinatal. Aproximadamente, $50 \%$ dos casos estão associados a outros defeitos congênitos não relacionados com o defeito básico da sirenomelia, como o do tubo neural e os cardíacos e anomalias do trato gastrointestinal superior ${ }^{(1,12-14)}$.

Relatamos três casos de sirenomelia associados a outras malformações raramente observadas, diagnosticados no Instituto Nacional de Saúde da Mulher, da Criança e do Adolescente Fernandes Figueira/Fundação Oswaldo Cruz (FIOCRUZ).

\section{Relato de casos}

\section{Caso 1}

Primigesta, 30 anos de idade, 23 semanas de gestação, com ultrassonografia mostrando adramnia, feto com fêmures curtos e higroma cístico. Casal não consanguíneo com história familiar de defeito de tubo neural. Gestante com hipertensão arterial crônica, em uso de inibidor de enzima conversora de angiotensina (ECA) no primeiro trimestre. Ressonância nuclear magnética (RM) revelou mielomeningocele e agenesia renal bilateral, não sendo identificados os membros inferiores. Interrupção da gestação na $29^{a}$ semana, após autorização judicial. O natimorto pesou $1.120 \mathrm{~g}$. À necropsia (Figuras 1 e 2), feto macerado com membro inferior único e rudimentar, ausência de genitália externa, mielomeningocele lombossacra, gastrosquise expondo alças intestinais, ânus imperfurado, agenesia renal bilateral, comunicação interventricular e artéria umbilical única. O exame radiológico do feto mostrou fêmur único, tíbia e fíbula ausentes (sirenomelia tipo VII, classificação de Stocker e Heifetz ${ }^{[15]}$ ).

\section{Caso 2}

Paciente de 28 anos, com diagnóstico ultrassonográfico de fêmur e artéria umbilical únicos na $12^{\mathrm{a}}$ semana de gestação. História familiar negativa para malformações. Sangramento vaginal de pequena monta na $15^{\mathrm{a}}$ semana. O estudo fetal detalhado por RM mostrou membro inferior único. Parto cesáreo na $30^{a}$ semana. $O$ recém-nascido pesou 1. 010 g, recebeu Apgar 1/4/7 e faleceu após 6 horas. A necropsia (Figura 3) mostrou, externamente, membro inferior único e rudimentar, ausência de genitália externa e ânus imperfurado. Internamente (Figuras 4 e 5), atresia do esôfago com fístula distal para traqueia, agenesia dos rins, dos ureteres e da bexiga, atresia retal e testículos abdominais. Cordão umbilical curto com única artéria. Ao exame radiológico (Figura 6), fêmur único e tíbia rudimentar única, classificada como tipo VI de Stocker e Heifetz ${ }^{(15)}$.

\section{Caso 3}

Gestante de 23 anos de idade, referida na $25^{\text {a }}$ semana com diagnóstico ultrassonográfico de adramnia, feto com membros inferiores fusionados, agenesia dos rins, dos ureteres e da bexiga. Terceira gestação de casal não consanguíneo, sem história familiar de malformações. Sangramento

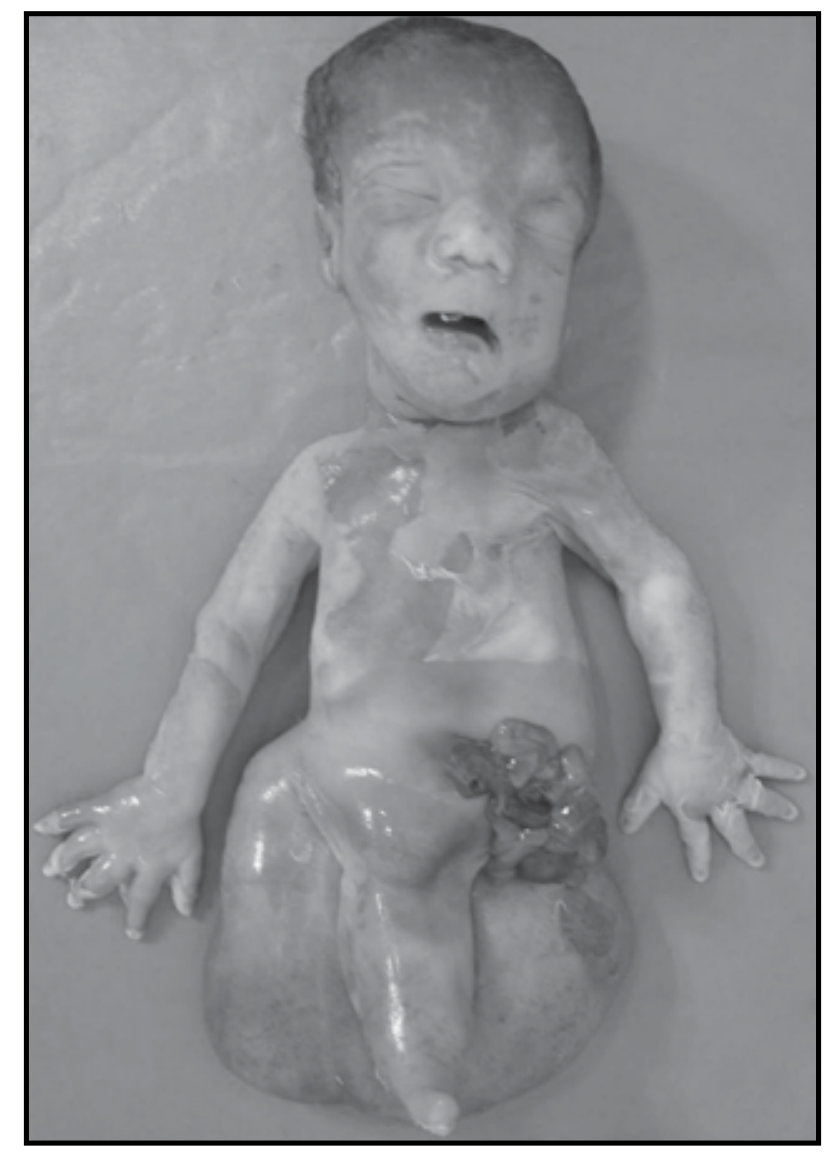

Figura 1 - Caso 1: feto macerado com membro inferior único rudimentar, agenesia de genitália externa, volumosa mielomeningocele lombossacra e gastrosquise expondo alças intestinais 


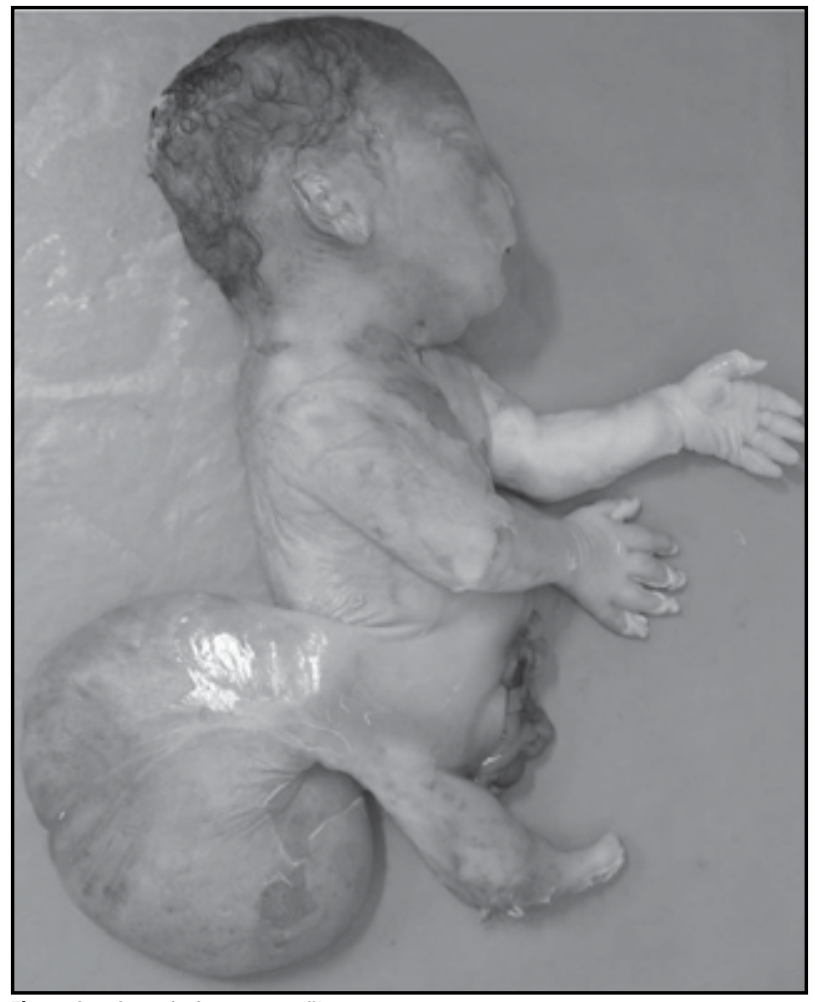

Figura 2 - Caso 1: feto em perfil

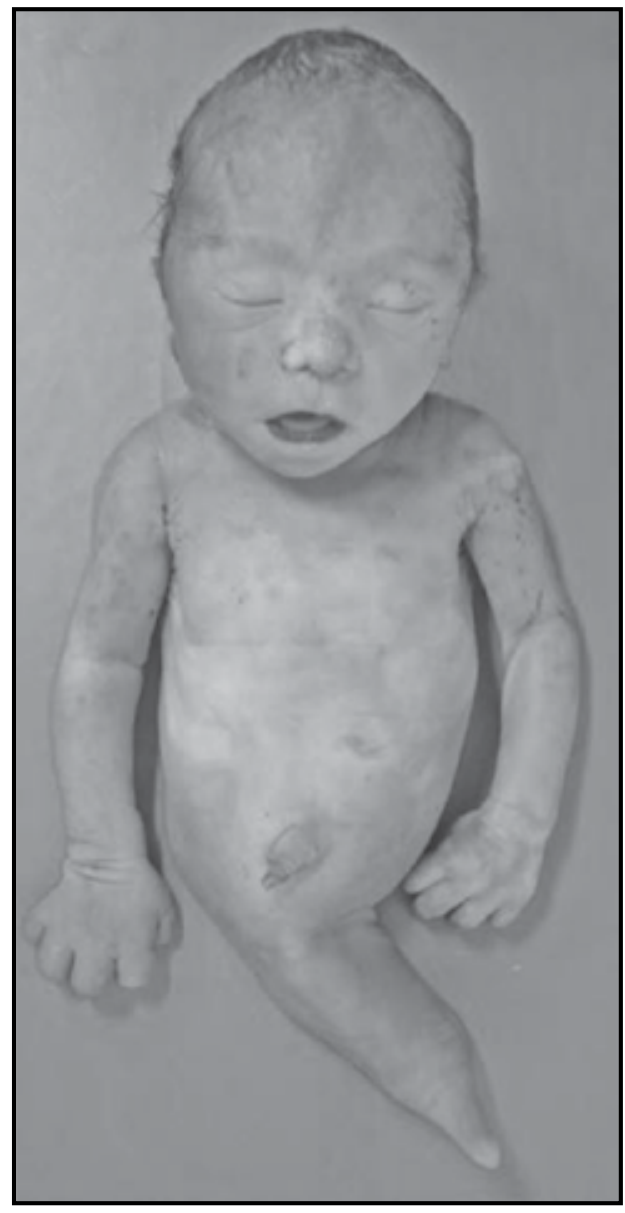

Figura 3 - Caso 2: recém-nascido com membro inferior único. Agenesia de genitália externa

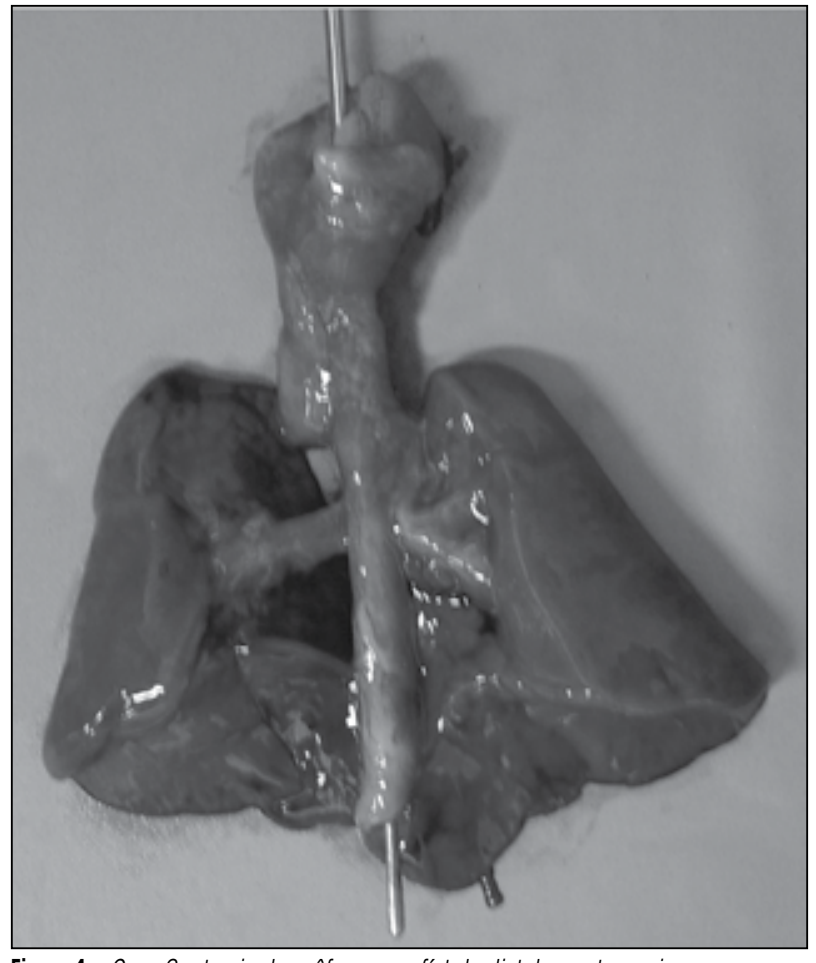

Figura 4 - Caso 2: atresia do esôfago com fístula distal para traqueia

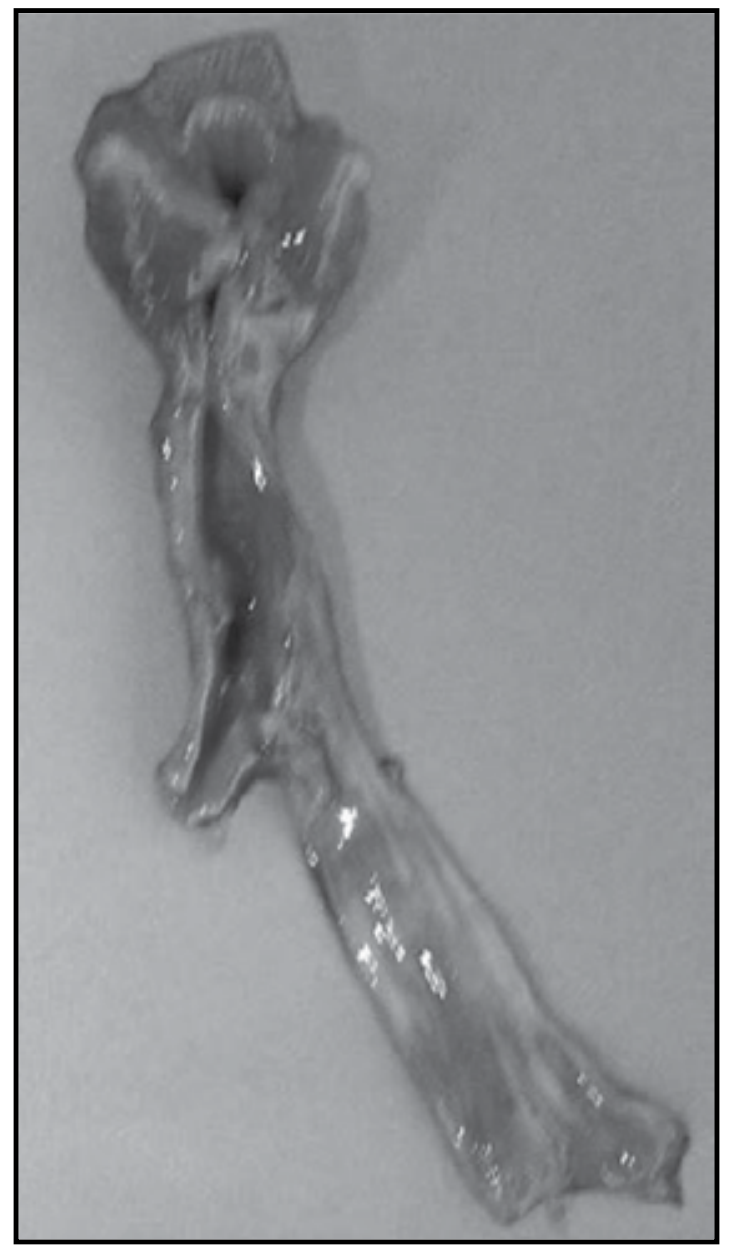

Figura 5 - Detalhe da figura anterior mostrando a fístula esôfago-traqueal 
vaginal na décima semana após exposição a misoprostol. Parto cesáreo a termo, recém-nascido com $2.010 \mathrm{~g}$ e óbito com 20 horas. A necropsia (Figura 7) evidenciou fenótipo de sirenomelia. Internamente, os achados ultrassonográficos de agenesia dos rins, dos ureteres e da bexiga foram confirmados, acrescidos de lobulação pulmonar invertida (Figura 8), comunicação interatrial, atresia do reto com imperfuração anal e artéria umbilical única. Genitália interna representada por testículos abdominais. O exame radiológico post mortem (Figura 9) mostrou fusão parcial dos fêmures, duas tíbias e uma fíbula, caracterizando o tipo IV de Stocker e Heifetz ${ }^{(15)}$.

\section{Discussão}

Sirenomelia é considerada um defeito do campo primário do desenvolvimento que afeta múltiplas estruturas primordiais da linha média ${ }^{(11)}$, podendo ter etiologia variada. Do ponto de vista etiopatogênico, foram aventadas duas hipóteses para explicar o desenvolvimento anormal dos membros inferiores ${ }^{(5,11)}$. A primeira relaciona-se com erro primário da blastogênese e a segunda, com evento vascular

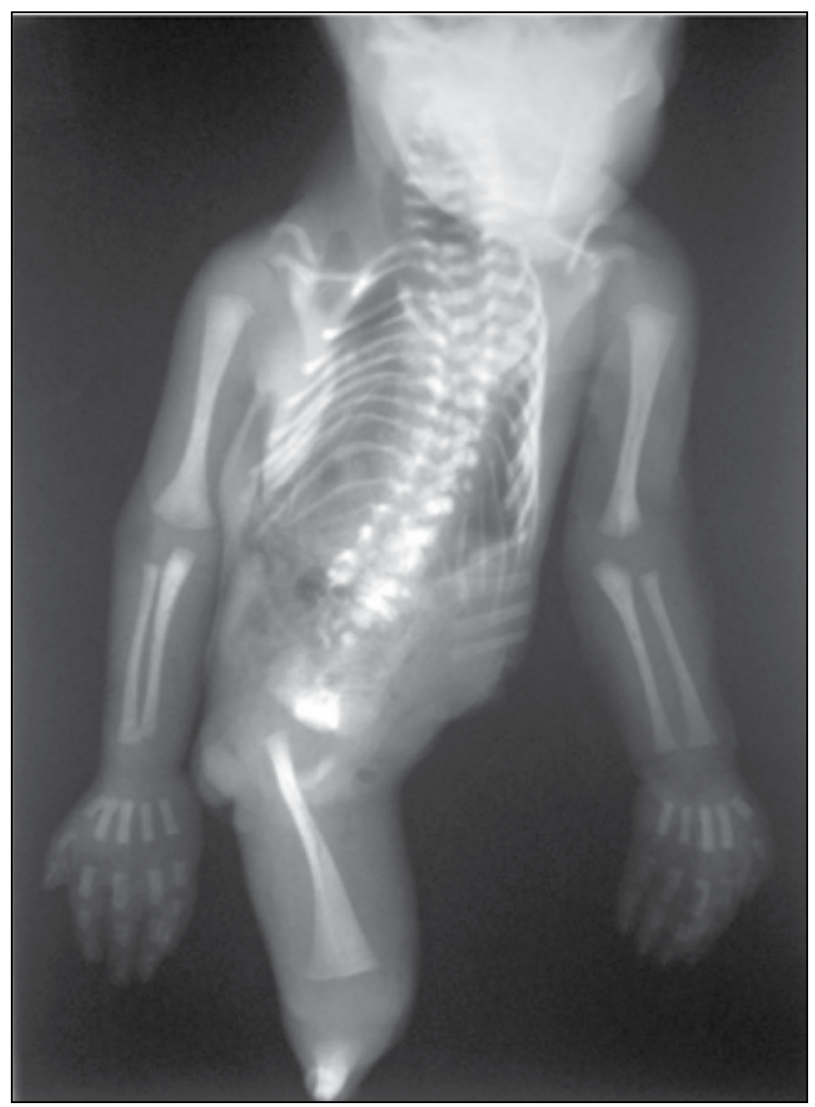

Figura 6 - Caso 2: radiografia mostrando fêmur único e tíbia rudimentar tipo VI de Sotcker e Heifetz ${ }^{(15)}$

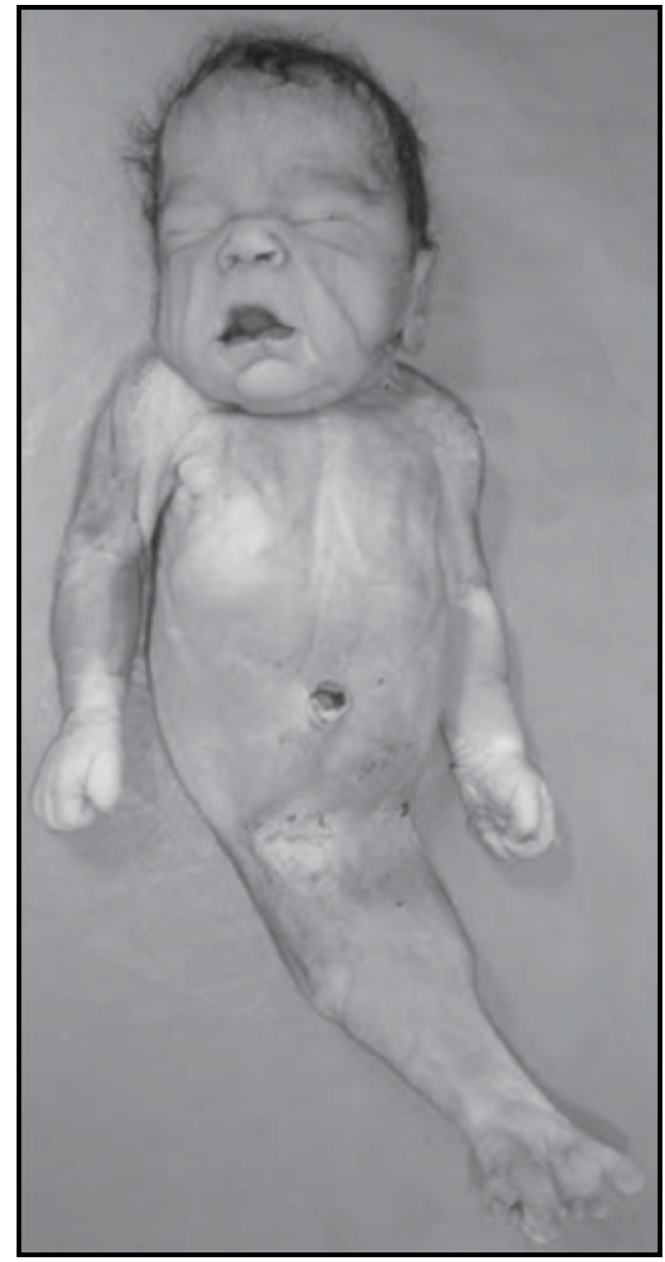

Figura 7 - Caso 3: recém-nascido a termo com membros inferiores fusionados e agenesia de genitália externa

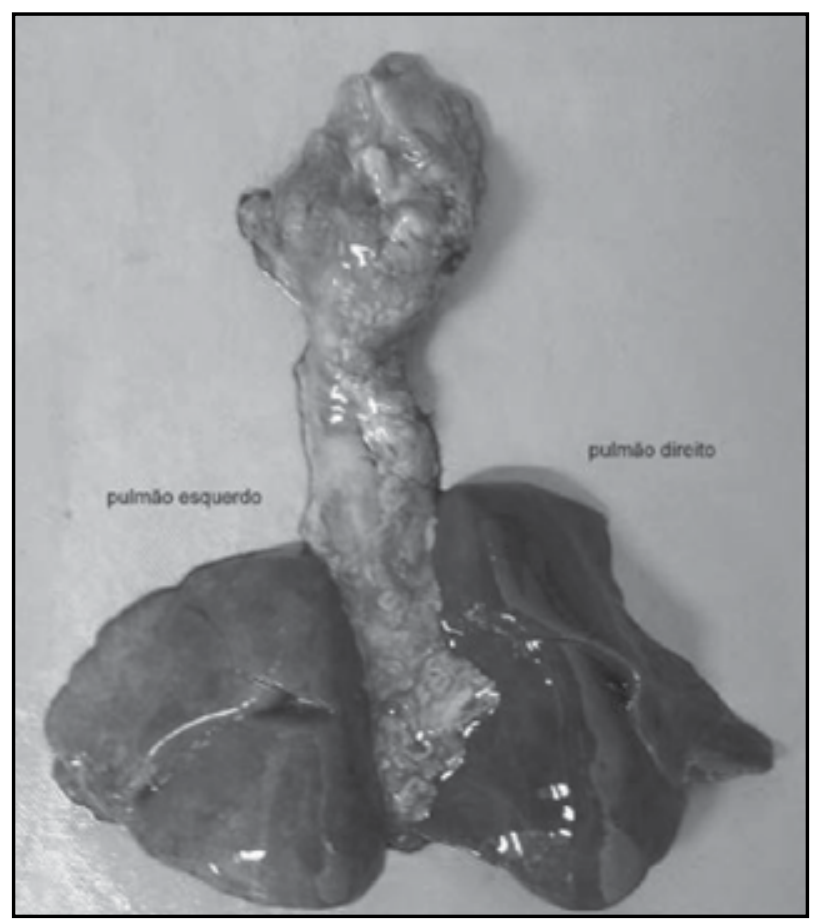

Figura 8 - Caso 3: pulmões com lobulação invertida 


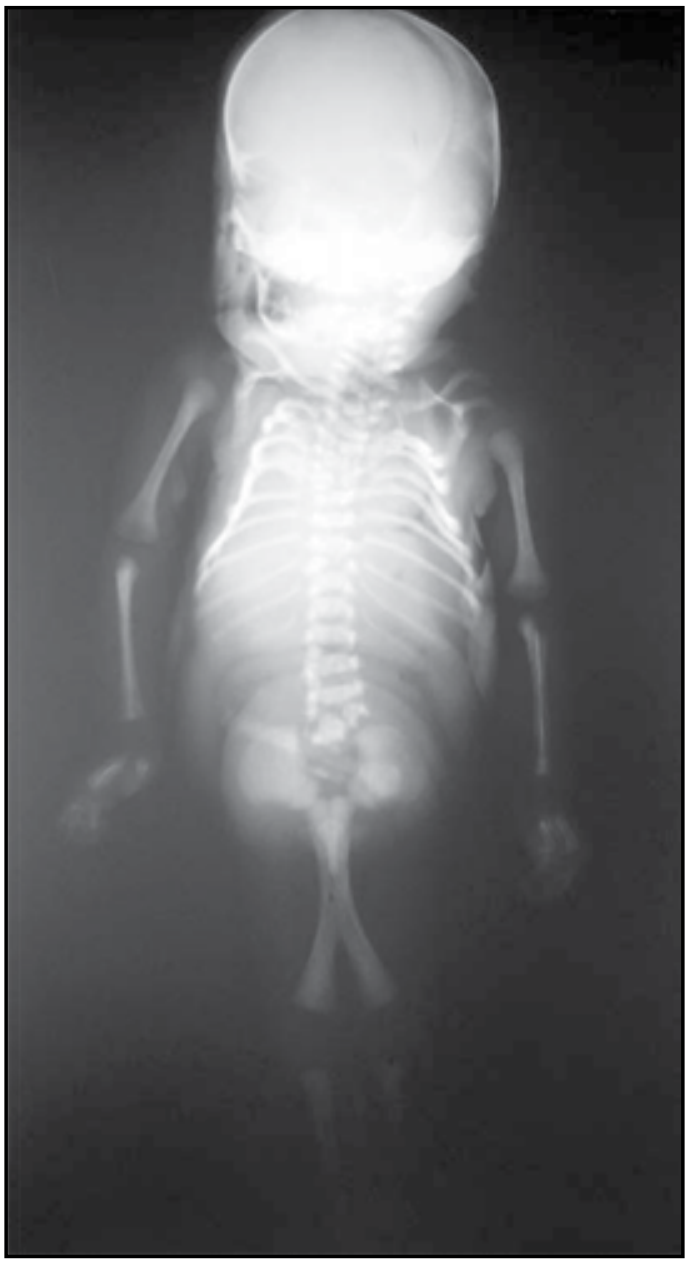

Figura 9 - Caso 3: radiografia mostrando fusão parcial dos fêmures, duas tíbias e uma fíbula tipo IV de Stocker e Heifetz ${ }^{(15)}$

com base no desenvolvimento anormal dos vasos umbilicais, resultando em aporte sanguíneo insuficiente para suprir a porção caudal do embrião(6). Garrido-Allepuz et al.(5) defendem que as duas teorias não se excluem mutuamente, embora a hipótese do defeito na blastogênese explique ambas as hipóteses. Esses mesmos autores ${ }^{(5)}$ referem uma possível base genética para explicar o fenótipo de sirenomelia em modelos experimentais, nos quais a deficiência da enzima Cyp26a1, que degrada o ácido retinoico, e a redução da proteína morfogenética do osso (pmo) afetariam o desenvolvimento normal da região caudal do embrião de camundongos. No entanto, até o momento, não há relatos em fetos humanos da associação da exposição ao ácido retinoico e à sirenomelia ${ }^{(5)}$.

Em 1987, Stocker e Heifetz ${ }^{(15)}$ classificaram os casos de sirenomelia, de acordo com a fusão e/ou agenesia dos ossos dos membros inferiores, em sete tipos. No tipo I, não há comprometimento numérico dos ossos, apenas fusão de partes moles, enquanto no tipo VII, está presente um fêmur único, não sendo observadas tíbia e fíbula. A classificação completa caracteriza-se da seguinte forma:

- tipo I - fêmures, tíbias e fíbulas em número habitual;

- tipo II - fíbula única fusionada;

- tipo III - ausência de fíbula;

- tipo IV - fêmures parcialmente fusionados e fíbula única;

- tipo V - fêmures parcialmente fusionados e fíbula ausente;

- tipo Vl: fêmur e tíbia únicos;

- tipo VII: fêmur único, tíbia e fíbula ausentes.

Na casuística ora apresentada, utilizamos essa classificação. No primeiro caso, embora defeitos cardíacos tenham sido relatados em associação à sirenomelia ${ }^{(3,12)}$, a exposição do feto aos inibidores da ECA poderia justificar a ocorrência da cardiopatia ${ }^{(2)}$. Os defeitos de fechamento da porção inferior do tubo neural podem estar patogenicamente associados, uma vez que a coluna lombossacra está comprometida na sirenomelia(13). Já a associação com gastrosquise é rara, tendo sido relatada por Mastroiacovo et al..$^{(10)}$. Feldkamp et al.(4) sugeriram que a gastrosquise seria um defeito primário e não um evento disruptivo, ocorrendo entre a terceira e a quinta semanas do desenvolvimento embrionário, mesmo período em que a sirenomelia supostamente ocorreria.

No segundo caso, a atresia do esôfago, outra associação rara, foi observada previamente por Duncan et al.(3) e é também exemplo de defeito do campo primário de desenvolvimento ${ }^{(9)}$.

No terceiro caso, a alteração da lobulação pulmonar pode ser considerada parte do fenótipo de situs inversus, igualmente ao outro defeito da blastogênese ${ }^{(9)}$.

Esses três casos representam exemplos de associação de defeitos do campo primário de desenvolvimento, raramente observados com sirenomelia, e somente puderam ter seus diagnósticos firmados por meio da necropsia perinatal.

A combinação do diagnóstico de imagem pré-natal e o estudo necroscópico são fundamentais para o estabelecimento do diagnóstico definitivo e a orientação, para o aconseIhamento genético, nos casos de malformação congênita. Em relação à sirenomelia, a maioria dos casos é esporádica, o que implica baixo risco de recorrência para as famílias. Por outro lado, é possível que a associação de outros defeitos do campo primário do desenvolvimento esteja sendo subestimada, uma vez que a ocorrência de sirenomelia na população é rara e, embora a fusão dos membros e a agenesia renal 
sejam rotineiramente diagnosticadas pela ultrassonografia, outras malformações associadas podem não ser detectadas. Assim, torna-se oportuno para melhor delinear o espectro de anomalias associadas à sirenomelia e, consequentemente, a investigação etiopatogênica desse defeito tão raro, a realização sistemática de necropsia dos afetados.

\section{Referências}

1. CHEN, C. P. et al. Sirenomelia with uncommon osseous fusion associated with a neural tube defect. Pediatr Radiol, v. 28, n. 5, p. 293-6, 1998.

2. COOPER, W. O. et al. Major congenital malformations after first-trimester exposure to ACE inhibitors. N Engl J Med, v. 354, n. 23, p. 2443-51, 2006.

3. DUNCAN, P. A.; SHAPIRO, L. R. Interrelationships of the hemifacial-microsomia-VATER, VATER and sirenomelia phenotypes. Am J Med Genet, v. 47, n. 1, p. 75-84, 1993.

4. FELDKAMP, M. L.; CAREY, J. C.; SADLER, T. W. Development of gastroschisis: review of hypotheses, a novel hypothesis, and implications for research. Am J Med Genet, v. 143A, n. 7, p. 639-52, 2007.

5. GARRIDO-ALLEPUZ, C. et al. A clinical and experimental overview of sirenomelia: insight into the mechanisms of congenital limb malformations. Dis Models Mech, v. 4, n. 3, p. 289-99, 2011.

6. HENTSCHEL, J. et al. Caudal regression sequence: vascular origin? J Perinatol, v. 26, n. 7, p. 445-7, 2006.

7. KÄLLEN, B. et al. The cyclops and the mermaid: an epidemiological study of two types of rare malformation. J Med Genet, v. 29, n. 1, p. 30-5, 1992.

8. LADURE, H. et al. Diagnostic antenatal d'une sirénomélie. J Gynecol Obstet Biol Reprod, v. 35, n. 2, p. 181-5, 2006.

9. MARTINEZ-FRIAS, M. L.; FRIAS, J. L. Primary developmental field III: clinical and epidemiological study of blastogenetic anomalies and their relationship to different MCD patterns. Am J Med Genet, v. 70, n. 1, p. 11-5, 1997.

10. MASTROIACOVO, P. et al. Gastroschisis and associated defects: an international study. Am J Med Genet, v. 143A, n. 7, p. 660-71, 2007.

11. OPITZ, J. M. et al. Defects of blastogenesis. Am J Med Genet, v. 115, n. 4, p. 269-86, 2002.

12. OPITZ, J. M.; WILSON, G. N.; GILBERT-BARNESS, E. Analysis of developmental pathology. In: Potter's Pathology of the fetus, infant and child. 2. ed. Philadelphia, PA: Mosby-Elsevier, 2007. Cap. 3, p. 97133.

13. ORIOLI, I. M. et al. Sirenomelia: an epidemiologic study in a large dataset from the International Clearinghouse of Birth Defects Surveillance and Research, and literature review. Am J Med Genet Part C Semin Med Genet, v. 157, n. 4, p. 358-78, 2011.

14. RODRIGUEZ, J. I.; PALACIOS, J. Craniorachischisis totalis and sirenomelia. Am J Med Genet, v. 43, n. 4, p. 732-6, 1992.

15. STOCKER, J. T.; HEIFETZ, S. A. Sirenomelia. A morphological study of 33 cases and review of the literature. Perspect Pediatric Pathol, v. 10, n. 10, p. 7-50, 1987. 